\title{
Nucleotide Sequence and Characterization of a Repetitive DNA Element from the Genome of Bordetella pertussis with Characteristics of an Insertion Sequence
}

\author{
By MARTHA A. MCLAFFERTY, * DAVID R. HARCUS AND \\ ERIK L. HEWLETT \\ Departments of Medicine and Pharmacology, University of Virginia School of Medicine, \\ Charlottesville, VA 22908, USA
}

(Received 24 February 1988)

\begin{abstract}
A repeating element of DNA has been isolated and sequenced from the genome of Bordetella pertussis. Restriction map analysis of this element shows single internal ClaI, SphI, Bst EII and SalI sites. Over 40 DNA fragments are seen in ClaI digests of $B$. pertussis genomic DNA to which the repetitive DNA sequence hybridizes. Sequence analysis of the repeat reveals that it has properties consistent with bacterial insertion sequence (IS) elements. These properties include its length of $1053 \mathrm{bp}$, multiple copy number and presence of $28 \mathrm{bp}$ of near-perfect inverted repeats at its termini. Unlike most IS elements, the presence of this element in the $B$. pertussis genome is not associated with a short duplication in the target DNA sequence. This repeating element is not found in the genomes of $B$. parapertussis or $B$. bronchiseptica. Analysis of a DNA fragment adjacent to one copy of the repetitive DNA sequence has identified a different repeating element which is found in nine copies in B. parapertussis and four copies in B. pertussis, suggesting that there may be other repeating DNA elements in the different Bordetella species. Computer analysis of the $B$. pertussis repetitive DNA element has revealed no significant nucleotide homology between it and any other bacterial transposable elements, suggesting that this repetitive sequence is specific for $B$. pertussis.
\end{abstract}

\section{INTRODUCTION}

Bacterial insertion sequence (IS) elements are discrete units of DNA which are capable of inserting themselves into multiple sites in a bacterial genome. The distinguishing properties of IS elements include their size (800-1800 bp), multiple copy number, presence of inverted repeats at their termini, and short duplication of target DNA at their insertion site. They also appear to encode only proteins that are involved in their own transposition (Calos \& Miller, 1980; Grindley \& Reed, 1985; Iida et al., 1983; Kleckner, 1981). This lack of encoded genetic markers, along with their small size, differentiates IS elements from larger bacterial transposons which encode phenotypically identifiable characteristics such as drug and antibiotic resistance. The exact function of IS elements is unknown, though they appear to be important for evolutionary processes by mediating chromosomal rearrangements such as deletions, inversions and duplications, and by altering expression of adjacent genes (Arber, 1983; Calos \& Miller, 1980; Rappuoli et al., 1987; Saesler et al., 1974; Scordilis et al., 1987). These elements were first identified in Escherichia coli but now have been found in numerous other prokaryotes.

Recently we have been analysing the genome of Bordetella pertussis in search of DNA fragments for use as pertussis-specific DNA probes. In the course of these studies we have found that this organism contains a sequence of DNA which is repeated many times throughout its genume, but is not present in B. parapertussis or B. bronchiseptica (McLafferty et al., 1986). This

Abbreviations: IPTG, isopropyl $\beta$-D-thiogalactoside; X-gal, 5-bromo-4-chloro-3-indolyl $\beta$-D-galactoside. 
observation has been substantiated by McPheat \& McNally (1987). With subsequent isolation and sequencing of this repeating DNA element from $B$. pertussis, we find that it has characteristics of bacterial IS elements.

\section{METHODS}

Bacterial strains, plasmids and growth conditions. B. pertussis Tohama has been described (Imaizumi et al., 1983). B. parapertussis CN8234 was obtained from P. Novotny (Wellcome Research Laboratories, Beckenham, UK). B. parapertussis 501 and B. bronchiseptica strains 213,361 and 469 are clinical isolates maintained in our laboratory. The bacteria were cultivated on Bordet-Gengou plates (Difco) or modified Stainer-Scholte broth (Hewlett \& Wolff, 1976). Cosmid p11-11 was obtained from A. A. Weiss (Medical College of Virginia, Richmond, VA, USA) and contains $B$. pertussis DNA cloned into cosmid pHC79. Specific restriction fragments of the cosmid p11-11 insert were cloned into plasmid pBR325 by standard procedures.

Isolation and manipulation of DNA. Plasmid DNA was isolated by the alkaline lysis procedure of Birnboim \& Doly (1979), and then purified by density centrifugation through a $\mathrm{CsCl} /$ ethidium bromide gradient (Maniatis $e t$ al., 1982). Genomic DNA was isolated by the method of Yee \& Inouye (1981). Restriction endonucleases were used according to the specifications of the manufacturer (New England BioLabs). DNA restriction fragments were resolved by electrophoresis through $0.9 \%$ agarose gels in Tris/acetate buffer (Maniatis et al., 1982). Specific DNA fragments were purified from the agarose gels using NA-45 DEAE paper (Schleicher \& Schuell) as described by the manufacturer.

DNA hybridization. The transfer of DNA from agarose gels to nylon membranes was done as recommended by the manufacturer (AMF Cuno). DNA probes were labelled with ${ }^{32} \mathrm{P}$ by nick-translation (Maniatis et al., 1982) and hybridized to the nylon membranes using the method of Church \& Gilbert (1984).

Preparation of DNA for sequencing. Fragments to be sequenced were cloned directly into M13mp19 or M13mp18 phage vectors, transfected into $E$. coli JM101 and plated onto YT plates (per litre: $8 \mathrm{~g}$ tryptone, $5 \mathrm{~g}$ yeast extract, $2.5 \mathrm{~g} \mathrm{NaCl}, \mathrm{pH} 7 \cdot 0$ ), containing X-gal and IPTG as recommended by the supplier (New England BioLabs). Each of the resulting colourless plaques was inoculated into $3 \mathrm{ml} \mathrm{YT}$ broth containing $30 \mu \mathrm{l}$ of an overnight culture of $E$. coli JM101. These cultures were incubated at $37^{\circ} \mathrm{C}$ with shaking at 400 r.p.m. for approximately $5 \mathrm{~h}$. The bacteria were then pelleted by centrifugation in a microfuge at 12000 r.p.m. for 15 min. From the resulting supernatants, $2 \mu \mathrm{l}$ was removed and spotted onto a nylon membrane and the remainder was purified for DNA sequencing. The nylon membranes were dried at room temperature and used in a hybridization reaction with the ${ }^{32}$ P-labelled DNA probes (Church \& Gilbert, 1984) to test for the presence of specific B. pertussis DNA inserts.

DNA sequencing. This was done by the dideoxy chain-termination method (Sanger et al., 1977) using reagents obtained from New England BioLabs. For sequencing inserts longer than approximately $300 \mathrm{bp}$, the Cyclone Biosystem (IBI) was used. Briefly, this system allows the construction of overlapping subclones from the DNA fragment cloned into the M13 vector through controlled deletions using T4 DNA polymerase. The final consensus sequence of the $B$. pertussis IS element was the result of sequencing each nucleotide at least twice in both directions.

Computer analysis. DNA sequence data were analysed using the programs of Conrad \& Mount (1984). The DNA sequences were compared using the FASTN program (Lipman \& Pearson, 1985), which searches the GenBank library for DNA homologies.

\section{RESULTS}

\section{Identification of the repeating DNA element in the genome of $B$. pertussis}

We have recently been studying the genome of $B$. pertussis to identify a fragment of DNA suitable for use as a pertussis-specific DNA probe. For these studies, we have used cosmid p11-11, which is one of a series of cosmid clones containing $B$. pertussis DNA generated by Dr Alison Weiss. Cosmid p11-11 was digested with ClaI and an $1100 \mathrm{bp}$ fragment was isolated and subcloned into plasmid pBR325. This 1100 bp fragment, designated Cla-4, was labelled with ${ }^{32} \mathrm{P}$ by nick-translation and hybridized to a Southern blot of ClaI digests of genomic DNA from $B$. pertussis, B. parapertussis and B. bronchiseptica (Fig. 1). It is evident from Fig. 1 that the 1100 bp Cla-4 probe contains a sequence of DNA which is repeated many times throughout the $B$. pertussis genome. There are at least 40 fragments which hybridize to the $\mathrm{Cla}-4$ probe in the $B$. pertussis Tohama strain. This 1100 bp fragment also hybridizes to DNA fragments from $B$. parapertussis and $B$. bronchiseptica, but there are fewer copies. There are nine fragments in $B$. parapertussis strains 501 and $\mathrm{CN} 8234$, ranging in size from 1 to $20 \mathrm{~kb}$, which hybridize to the Cla-4 probe, and these fragments show the same chromosomal distribution in both strains. 


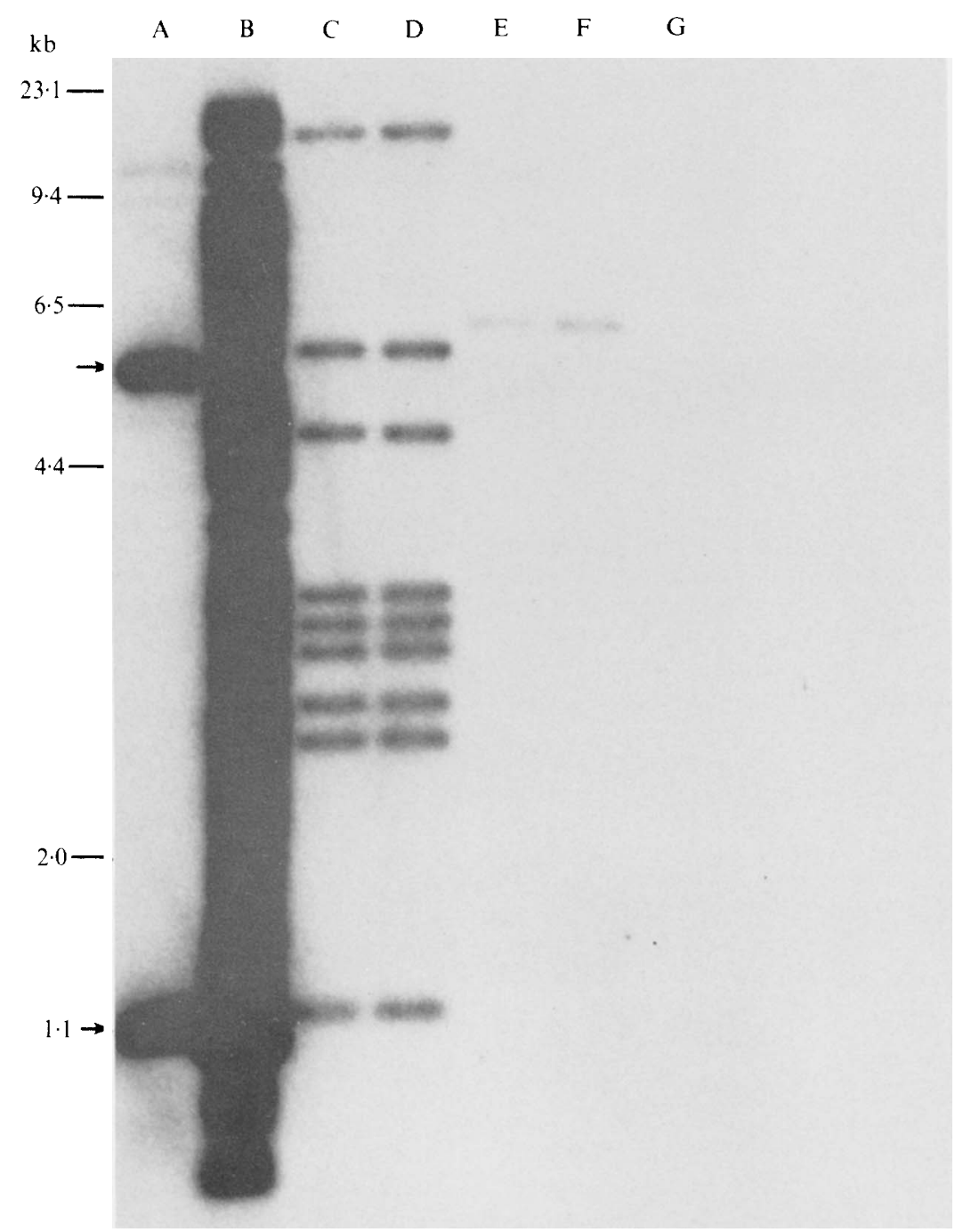

Fig. 1. Southern blot of ClaI-digested genomic DNA from Bordetella species hybridized to the $1100 \mathrm{bp}$ Cla-4 probe, showing the presence of the repeating sequence. Lanes: A, ClaI digest of cosmid p11-11, with arrows marking the $1100 \mathrm{bp} \mathrm{Cla}-4$ probe and the $5 \mathrm{~kb} \mathrm{Cla}-3$ fragment which also hybridizes to the Cla-4 probe; B, B. pertussis Tohama; C, B. parapertussis CN8234; D, B. parapertussis 501; E, B. bronchiseptica $213 ;$ F, B. bronchiseptica $361 ;$ G, B. bronchiseptica 469.

B. bronchiseptica strains 361 and 213 each contain one band of approximately $6 \mathrm{~kb}$ which hybridizes to the Cla-4 probe. There is no hybridization of the Cla-4 probe to DNA from B. bronchiseptica strain 469 .

\section{Isolation of the repeating element}

To identify the complete repeating element, it was necessary to have at least two different copies of it for sequence comparison. This enabled the boundaries of the element to be accurately determined. Since the $1100 \mathrm{bp} \mathrm{Cla}-4$ fragment hybridizes to a $5 \mathrm{~kb}$ fragment also obtained from the ClaI digest of cosmid p11-11, this indicates that the $5 \mathrm{~kb}$ fragment is likely to contain at least one copy of the repeating element (Fig. 1). A comparison of the $5 \mathrm{~kb}$ fragment (designated $\mathrm{Cla}-3$ ) and the $\mathrm{Cla}-4$ sequences should thus identify the repeating element. To test this hypothesis, the entire Cla-4 fragment was cloned into M13mp19 and sequenced. Restriction 


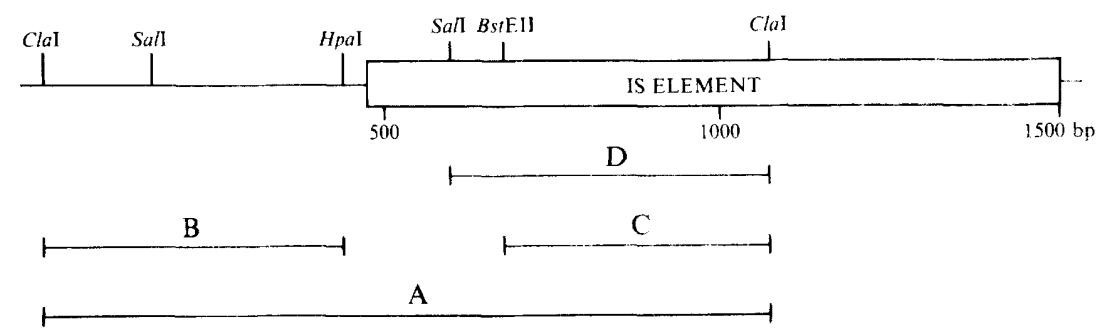

Fig. 2. Restriction map of the $1100 \mathrm{bp} \mathrm{Cla}-4$ probe and its position with respect to the $B$. pertussis repetitive DNA element. Fragment A is the 1100 bp Cla-4 fragment. Fragment D (SalI-ClaI fragment) and fragment $\mathrm{C}$ (BstEII-ClaI fragment) are internal to the repeating element. Fragment B ( $\mathrm{HpaI}-\mathrm{ClaI}$ fragment) is external, but adjacent to the $B$. pertussis repetitive DNA element and contains the repeating sequence found in $B$. parapertussis. Restriction fragments $B, C$ and $D$ are all derived from the Cla-4 fragment.

site analysis of the Cla-4 sequence shows two internal SalI sites and one internal SphI site. Because the SalI and SphI sites were believed to be within the repeating element, these two enzymes were used to digest the $5 \mathrm{~kb} \mathrm{Cla}-3$ fragment and thus increase the likelihood of obtaining matching sequences. The fragments of Cla-3 obtained from these digests were shotgun cloned into the SalI and SphI sites of M13mp19 and the resulting colourless plaques which hybridized to the Cla-4 probe were picked and sequenced. From a comparison of the sequence data, the $5^{\prime}$ terminus of the repeating element was determined and it showed that there are approximately 465 nucleotides of the $\mathrm{Cla}-4$ fragment which precede the start of the repeating element.

\section{Determination of the $3^{\prime}$ terminus of the repeating element}

Sequence comparison of the Cla-3 and Cla-4 restriction fragments was not adequate to identify the entire repeating sequence, due to the presence of a ClaI site in the middle of the repeating element (Fig. 2). To obtain the sequence of the repeat to its $3^{\prime}$ terminus, the cosmid p11-11 was digested with SalI. The SalI fragments were shotgun cloned into M13mp19 and the resulting colourless plaques were screened by plaque hybridization to a $460 \mathrm{bp} \mathrm{SalI-ClaI}$ fragment isolated from the $1100 \mathrm{bp} \mathrm{Cla}-4$ probe (Fig. 2, fragment D). This $460 \mathrm{bp}$ fragment was chosen for a probe since it contains DNA only within the repeating element. Using this method, three clones were identified and sequenced and the $3^{\prime}$ terminus of the repeating element was determined from the termination of sequence homology among the three fragments.

\section{Sequence analysis}

The entire DNA sequence of the repeating element identified in B.pertussis is shown in Fig. 3. The size of the repeating element is $1053 \mathrm{bp}$ and its termini (underlined in Fig. 3) consist of $28 \mathrm{bp}$ of near-perfect inverted repeats. These termini may hybridize to form the double-stranded stemloop structure shown in Fig. 4. This repetitive DNA element has a $\mathrm{G}+\mathrm{C}$ base content of $63.7 \%$ $(19.1 \% \mathrm{~A}, 17.2 \% \mathrm{~T}, 28.1 \% \mathrm{G}, 35.6 \% \mathrm{C})$, which is consistent with the high $\mathrm{G}+\mathrm{C}$ content reported for $B$. pertussis DNA (Locht \& Keith, 1986). The terminal inverted repeats of the $B$. pertussis repetitive DNA element have a much higher A $+\mathrm{T}$ base content $(64.3 \% \mathrm{~A}+\mathrm{T})$ than the rest of the element. This is similar for other IS elements, where the ends of the element contain a 3-13\% higher percentage of $\mathrm{A}-\mathrm{T}$ base pairs than the elements themselves (Kleckner, 1981).

\section{Analysis of the target DNA flanking the insertion site}

Another characteristic of bacterial insertion sequence elements is that they form short duplications in the target DNA at the insertion site (Calos \& Johnsrud, 1978; Calos \& Miller, 1980 ). To determine whether this occurred with the $B$. pertussis repetitive DNA element, we isolated one entire copy so that both the terminal inverted repeats as well as the flanking DNA 
CTAGGTGTGAAGATTCAATAGGTTGTATGCATGGTTCATCCGAACCGGATTTGAGAAACT

SphI

GGAAATCGCCAACCCCCCAGTTCACTCAAGGAGCCCGGCCGGATGAACACCCATAAGCAT Sall GCCCGATTGACCTTCCTACGTCGACTCGAAATGGTCCAGCAATTGATCGCCCATCAAGTT BstEII TGTGTGCCTGAAGCGGCCCGCGCCTATGGGGTCACCGCGCCGACTGTGCGCAAATGGCTG GGCCGCTTCCTGGCTCAGGGCCAGGCGGGCTTGGCCGATGCGTCCTCGCGCCCGACGGTC TCGCCCCGAGCGATTGCGCCGGCCAAGGCGCTGGCTATCGTGGAGCTGCGCCGCAAGCGG

CTGACCCAAGCGCGCATCGCCCAGGCGCTGGGCGTGTCAGCCAGCACCGTCAGCCGCGTC CTGGCCCGCGCCGGTCTGTCGCACCTGGCCGACCTGGAGCCGGCCGAGCCGGTGGTGCGC TACGAGCATCAGGCCCCCGGCGATCTGCTGCACATCGACATCAAGAAGCTGGGACGTATC CAGCGCCCTGGCCACCGGGTCACGGGCAACCGACGCGATACCGTTGAGGGGGCCGGCTGG $\mathrm{ClaI}$ GACTTCGTCTTCGTGGCCATCGATGACCACGCCCGCGTGGCCTTCACCGACATCCACCCC GACGAGCGCTTCCCCAGCGCCGTCCAGTTCCTCAAGGACGCAGTGGCCTACTACCAGCGC CTGGGCGTGACCATCCAGCGCTTGCTCACCGACAATGGCTCGGCCTTTCGCAGCCGCGCC TTCGCCGCGCTGTGCCATGAGCTGGGCATCAAGCACCGCTTTACCCGACCTTACCGCCCA CAGACCAATGGCAAGGCCGAACGCTTCATCCAGTCGGCCTTGCGTGAGTGGGCTTACGCT CACACCTACCAGAACTCCCAACACCGAGCCGATGCCATGAAATCCTGGCTACACCACTAC AACTGGCATCGACCCCACCAAGGCATCGGGCGCGCTGTACCCATCTCCAGACTCAACCTG GACGAATACAACCTATTGAATCTTCACAGCTAG

Fig. 3. Complete nucleotide sequence of the putative $B$. pertussis IS element. The sequence is written $5^{\prime}-3^{\prime}$, with the relevant restriction sites underlined and labelled. The $28 \mathrm{bp}$ terminal inverted repeats are underlined and mark the boundaries of the element.

could be examined. Since restriction site analysis shows that there are no internal BamHI or Eco RI sites, cosmid p11-11 was digested with BamHI and a $3.5 \mathrm{~kb}$ fragment containing one copy of the repeating element was cloned into M13mp19 and sequenced. As shown in Fig. 4, the target DNA flanking the $B$. pertussis repeating element was not duplicated during the integration process.

Absence of the B. pertussis repetitive DNA element in B. parapertussis and B. bronchiseptica

Since the Cla-4 probe used in the hybridization studies with genomic DNA of Bordetella species contains DNA from the repetitive sequence as well as approximately 465 bp of adjacent DNA, it was unclear whether the repetitive element itself is present in all of the Bordetella species or whether the flanking $B$. pertussis DNA is responsible for the hybridization to $B$. parapertussis and $B$. bronchiseptica DNA. To distinguish between these possibilities, a 365 bp $B s t$ EII-ClaI fragment internal to the $B$. pertussis repetitive sequence (Fig. 2, fragment C), and a $440 \mathrm{bp} \mathrm{HpaI-ClaI}$ fragment outside the repetitive sequence (Fig. 2, fragment B), were isolated 


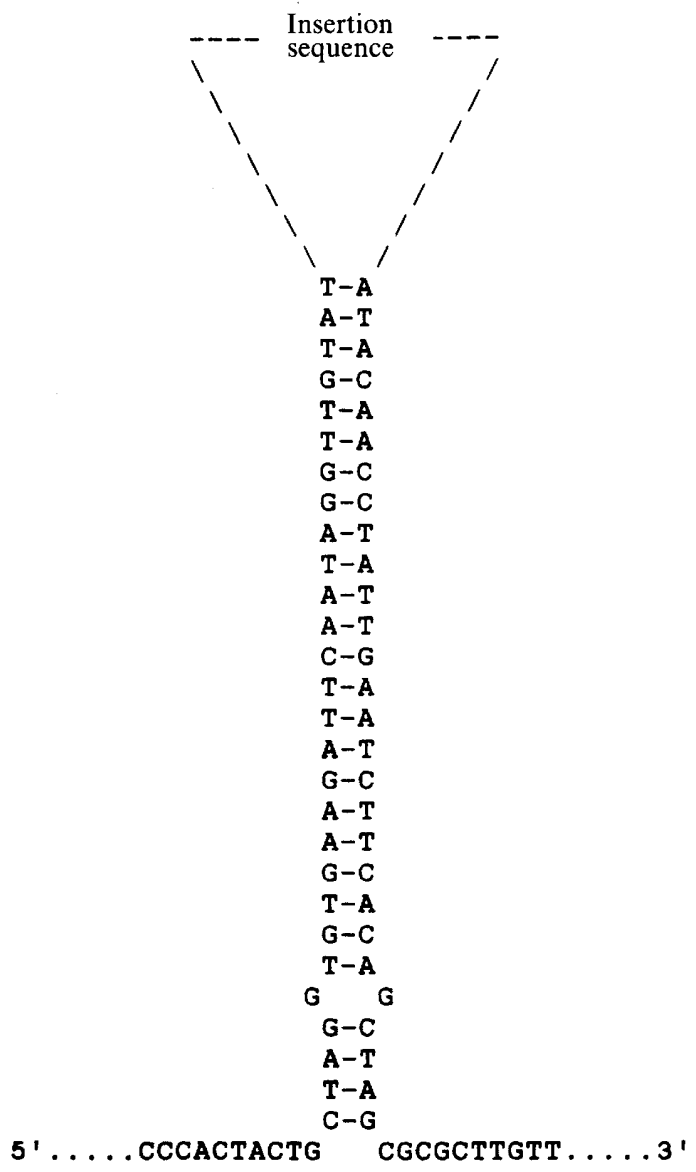

Fig. 4. Nucleotide sequence of the termini of the $B$. pertussis repetitive DNA element. The termini are near-perfect inverted repeats which may hybridize to form the double-stranded, stem-loop structure shown in this figure. Sequence data from other copies of this element show that the $\mathrm{G}$ and $\mathrm{C}$ nucleotides flanking it are not actually part of the element.

from the 1100 bp Cla-4 fragment (Fig. 2, fragment A). These two different fragments were hybridized to identical Southern blots containing ClaI digests of genomic DNA from $B$. pertussis, $B$. parapertussis and $B$. bronchiseptica. The results, shown in Fig. 5, indicate that fragment $C$, internal to the $B$. pertussis repetitive element, is specific for $B$. pertussis and is not found in $B$. parapertussis. A $6 \mathrm{~kb}$ fragment of $B$. bronchiseptica DNA hybridizes to fragment $C$ (Fig. $5 a$, lanes D and E), but this fragment does not appear to be a copy of the repetitive DNA element due to its absence when different internal fragments of the element are hybridized to $B$. bronchiseptica DNA (data not shown). The HpaI-ClaI fragment B, external but adjacent to the $B$. pertussis repetitive DNA sequence, hybridizes to nine fragments in $B$. parapertussis, four fragments in $B$. pertussis and none in B. bronchiseptica (Fig. $5 b$ ). This fragment accounts for the hybridization to $B$. parapertussis DNA seen with the Cla-4 probe (Fig. 1) and suggests that there is another, different repeating sequence in $B$. parapertussis with a few copies in $B$. pertussis. Further characterization of this fragment will determine whether it is also a bacterial IS element.

Computer analysis of the $B$. pertussis repetitive DNA sequence

The $B$. pertussis repetitive DNA sequence was examined for nucleotide homology with other known prokaryotic transposable elements (including IS elements) and no significant homology was found. The DNA sequence was also translated in all six reading frames to identify potential 
(a)

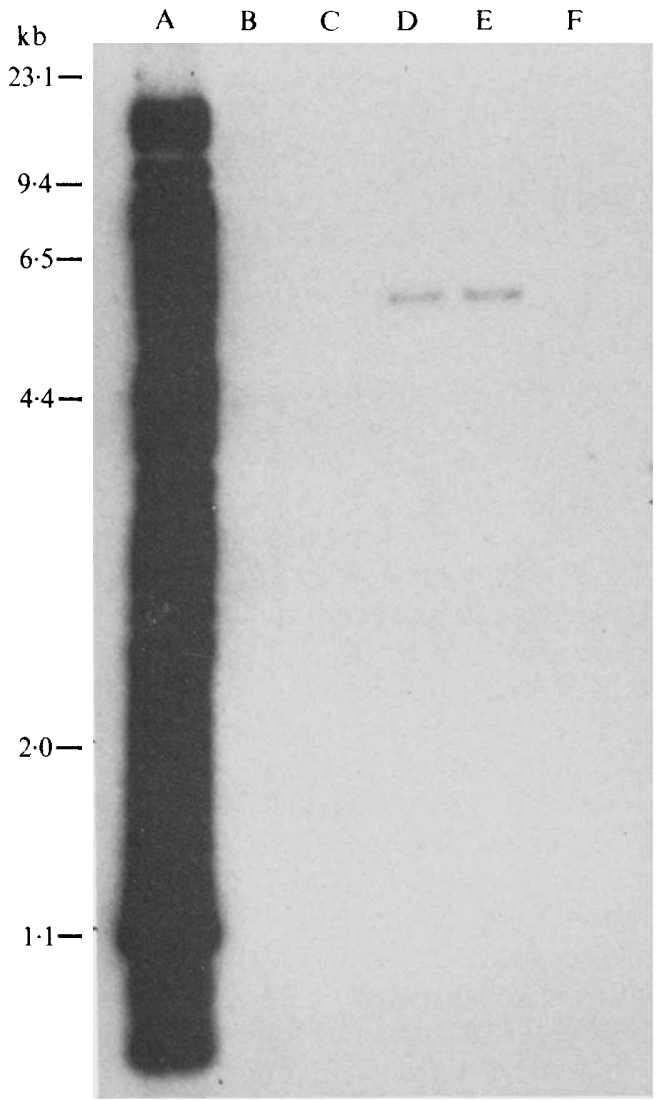

(b)

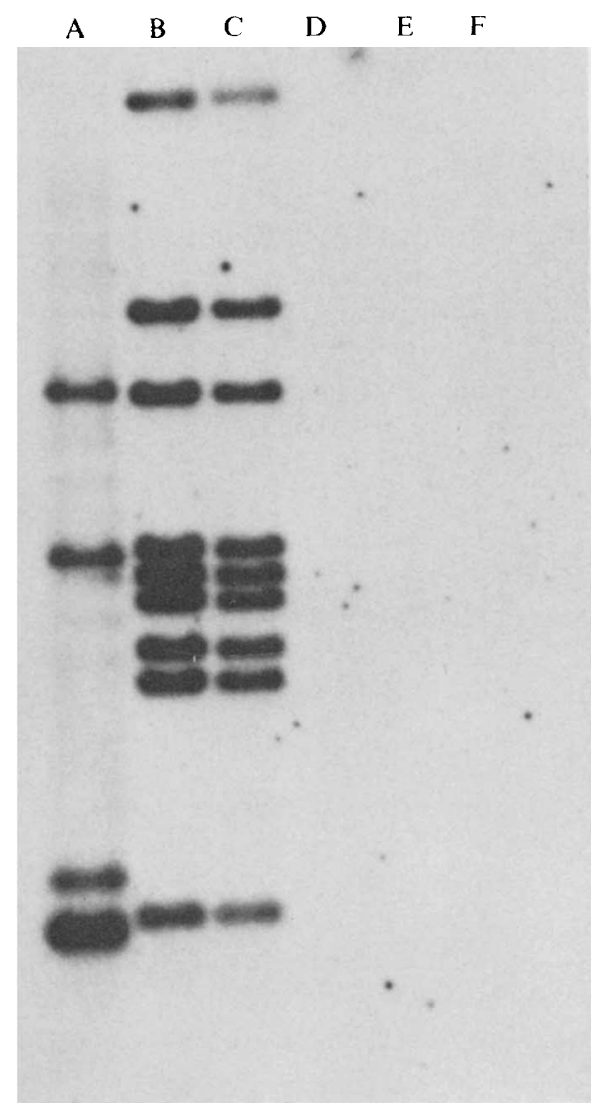

Fig. 5. Southern blots of ClaI-digested genomic DNA of Bordetella species hybridized to (a) a $365 \mathrm{bp}$ BstEII-ClaI fragment (shown in Fig. 2, fragment C), internal to the repeating element, or (b) a $440 \mathrm{bp}$ HpaI-ClaI fragment (shown in Fig. 2, fragment B), external to the repeating element. Lanes: A, B. pertussis Tohama; B, B. parapertussis CN8234; C, B. parapertussis 501 ; D, B. bronchiseptica 213 ; E, B. bronchiseptica 361; F, B. bronchiseptica 469 .

protein-coding regions, and several open reading frames (ORFs) were found. One large ORF extended from nucleotide 234 to 585 and would code for a polypeptide of 117 amino acids. Another ORF was found on the opposite strand running from nucleotides 256 to 654 and would code for a polypeptide of 133 amino acids. These ORFs are similar to those found with other bacterial IS elements (Kleckner, 1981; Rasmussen et al., 1987; Timmerman \& Chen-Pei, 1985), but are generally shorter in length. It is not known whether these ORFs code for functional proteins, although one or both could code for a transposase like those found with other IS elements (Grindley \& Reed, 1985).

\section{DISCUSSION}

We have identified and sequenced a segment of DNA from $B$. pertussis that is $1053 \mathrm{bp}$ long and is found in multiple copies in the $B$. pertussis genome. This sequence has properties consistent with bacterial IS elements, including its size, multiple copy number and presence of $28 \mathrm{bp}$ terminal inverted repeats. It does not appear that the integration of this element into the $B$. pertussis genome results in a short duplication of the target DNA at the insertion site.

Duplication of the target DNA at the IS insertion site is a common feature of IS elements and the only other IS element known to lack this property is IS91 (Diaz-Aroco et al., 1987). A 
comparison of IS 91 and the $B$. pertussis repetitive DNA sequence shows that the ends of both elements are defined by restriction sites. There are TaqI sites (TCGA) at the ends of IS $9 I$ and MaeI sites (CTAG) at the ends of the $B$. pertussis element. It appears that having restriction sites at the ends of an IS element is novel, and that it may influence the site of insertion as well as the mechanism by which the element inserts. It is possible, however, that these restriction sites are actually part of the target DNA rather than the IS element. Since these restriction sites are palindromes they could either hybridize to each other to be part of the terminal inverted repeats of the IS element, or could be the direct repeats (target DNA duplications) normally formed upon insertion of the IS element. If these restriction sites were part of the target DNA rather than the IS element, it would suggest that IS 91 and this $B$. pertussis IS element insert specifically at TaqI or MaeI sites in the target DNA. Diaz-Aroco et al. (1987) have shown, though, that the TaqI sites are actually part of the IS 91 sequence and the IS 91 does not insert specifically at TaqI sites in the target DNA. They suggest that the lack of target DNA duplications with insertion of IS 91 may be explained by the method of insertion. Currently it is hypothesized that IS elements insert by creating a staggered cleavage in the target DNA at the insertion site. The short target DNA duplications would form by repair of the staggered ends for ligation with the IS element. Diaz-Aroco et al. (1987) suggest that transposition of IS 91 may produce a blunt rather than a staggered cleavage in the target DNA, and thus would not produce the duplications usually seen. The similarity between the ends of IS 91 and the putative $B$. pertussis insertion element suggests that their mechanisms of integration into the target DNA may be the same. Further research into the actual transposition of this $B$. pertussis DNA element should clarify the role, if any, of these terminal restriction sites in the transposition of this insertion sequence.

Another property of the $B$. pertussis repetitive DNA element is that it is found in many copies in the $B$. pertussis genome. This large copy number is uncommon for IS elements in general but has been reported for Shigella species, which have 30-40 copies of insertion element ISI (Nyman et al., 1981). We also report the absence of this repetitive DNA element in B. parapertussis and $B$. bronchiseptica genomic DNA. These results are similar to those of McPheat \& McNally (1987), who reported a single band of $1.45 \mathrm{~kb}$ in genomic digests of $B$. parapertussis strain BPAH 1 and two bands in genomic digests of $B$. bronchiseptica strain BBRH 1 which hybridized to a $B a m H I$ fragment probe that contained a repeating sequence of $B$. pertussis DNA. This hybridization to $B$. parapertussis and $B$. bronchiseptica DNA is most likely due to the presence of DNA external to the repeating sequence and therefore suggests that we have isolated the same repetitive DNA sequence. Also, the size of approximately $1.0 \mathrm{~kb}$ and presence of one internal ClaI site reported by McPheat \& McNally (1987) for the repeating sequence they identified is consistent with our results. However, the fact that we have identified another repeating sequence present in nine copies in B. parapertussis and four copies in B. pertussis suggests that more than one kind of repetitive DNA sequence may be present in Bordetella species. Other bacteria, such as $E$. coli, possess several different types of IS elements (Calos \& Miller, 1980). It is also possible that different strains of $B$. pertussis have different copy numbers of this repeating sequence. We have tested eight different clinical isolates of $B$. pertussis, and have found them all to contain approximately the same number of copies of the repeating sequence (data not shown). None of the three strains of $B$. parapertussis or four strains of $B$. bronchiseptica that we have examined contain the $B$. pertussis insertion sequence. Study of additional strains of Bordetella species will determine whether there is consistency in the copy number of the repeating element as well as identify other possible repetitive DNA sequences.

The exact function of IS elements is unknown, but they appear to play an important evolutionary role by mediating chromosomal rearrangements and altering expression of adjacent genes, which may help organisms adapt to new environmental conditions (Arber, 1983; Syvanen, 1984). Examination of Bordetella species by multilocus enzyme electrophoresis has shown $B$. bronchiseptica to be the ancestral strain from which B. parapertussis and B. pertussis evolved and also that $B$. parapertussis is more closely related to $B$. bronchiseptica than to $B$. pertussis (Musser et al., 1986). If the putative IS element is indeed involved in evolutionary processes, then it is interesting to note the large copy number of this element in the most evolutionarily advanced species. This suggests that the element plays an important functional 
role for $B$. pertussis and may be aiding in its adaptation to new environmental conditions by controlling such things as phase variation and thus production of virulence determinants. Transposable elements have been found to activate gene expression in Pseudomonas cepacia (Scordilis et al., 1987) and E. coli, and to inactivate diphtheria toxin production in Corynebacterium diphtheriae (Rappuoli et al., 1987). Therefore, it is conceivable that this element is involved in gene expression in $B$. pertussis.

Even though the exact function of this IS element is unknown, its properties suggest that it might be useful for studying the epidemiology of pertussis infection. The large copy number of this element indicates that it would be a sensitive diagnostic probe for detecting $B$. pertussis organisms in clinical specimens. In fact, we find that the ${ }^{32} \mathrm{P}-$ labelled $\mathrm{Cla}-4$ probe, which contains a portion of the $B$, pertussis IS element as well as the repeating sequence found in $B$. parapertussis, is able to detect $10^{3} \mathrm{~B}$. pertussis organisms and $10^{5} \mathrm{~B}$. parapertussis organisms filtered onto nylon membranes (McLafferty et al., 1986). The fact that this repetitive DNA sequence may be species specific, as evidenced by its lack of significant homology with other known bacterial DNA sequences, suggests that it would be a specific as well as a sensitive tool for use in diagnosis of $B$. pertussis infection. We propose to designate this newly characterized $B$. pertussis IS element IS481 (Lederberg, 1987).

The authors gratefully acknowledge the assistance of the following individuals in this work: Miss Cynthia Kogut, and Mr Jonathan Skinner for preparation of the manuscript; Dr Pavel Novotny for supplying Bordetella strains; Dr Alison Weiss for providing cosmid p11-11; and Dr Kevin Lynch and Dr Robert Kadner for important discussion and suggestions. This work was conducted with the support of a contract from the Centers for Disease Control (DHHS 200-84-0784), a grant from the National Institutes of Health (AI18000) and the facilities of the University of Virginia Diabetes Center Molecular Biology Core Laboratory (supported by NIH grant AM22125).

\section{REFERENCES}

ARBER, W. (1983). Bacterial 'inserted sequence' elements and their influence on genetic stability and evolution. Progress in Nucleic Acid Research and Molecular Biology 29, 27-33.

Birnboim, H. C. \& Doly, J. (1979). A rapid alkaline extraction procedure for screening recombinant plasmid DNA. Nucleic Acids Research 7, 1513-1524.

Calos, M. P. \& JohnsRud, L. (1978). DNA sequence at the integration sites of the insertion element ISI. Cell 13, 411-418.

Calos, M. P. \& Miller, J. H. (1980). Transposable elements. Cell 20, 579-595.

Church, G. M. \& GilberT, W. (1984). Genomic sequencing. Proceedings of the National Academy of Sciences of the United States of America 81, 1991-1995.

ConRad, B. \& Mount, D. W. (1984). Microcomputer programs for DNA sequence analysis. Nucleic Acids Research 10, 31-38.

Diaz-Aroco, E., Mendiola, M. V., Zabala, J. C. \& DE LA CRUZ, F. (1987). Transposition of IS91 does not generate a target duplication. Journal of Bacteriology 169, 442-443.

GRINDLeY, N. D. F. \& ReED, R. R. (1985). Transpositional recombination in prokaryotes. Annual Review of Biochemistry 54, 863-896.

HewLeTt, E. \& WolfF, J. (1976). Soluble adenylate cyclase from the culture medium of Bordetella pertussis: purification and characterization. Journal of Bacteriology 127, 890-898.

IIDA, S., MEYER, J. \& ARBER W. (1983). Procaryotic IS elements. In Mobile Genetic Elements, pp. 159-221. Edited by J. A. Shapiro. London: Academic Press.
Imaizumi, A., Suzuki, Y., ONo, S., Sato, H. \& Sato, $Y$. (1983). Effect of heptakis-(2,6- $O$-dimethyl)- $\beta$ cyclodextrin on the production of pertussis toxin by Bordetella pertussis. Infection and Immunity 41, 1138-1143.

KLECKNER, N. (1981). Transposable elements in prokaryotes. Annual Review of Genetics 15, 341-404.

LEDERBERG, E. M. (1987). Plasmid reference center registry of transposon ( $\mathrm{Tn}$ ) and insertion sequence (IS) allocations through December 1986. Gene 51, 115-118.

Lipman, D. J. \& Pearson, W. R. (1985). Rapid and sensitive protein similarity searches. Science 227, 1435-1441.

LOCHT, C. \& KeITH, J. M. (1986). Pertussis toxin gene: nucleotide sequence and genetic organization. Science 232, 1258-1264

Maniatis, T., Fritsch, E. F. \& Sambrook, J. E. (1982). Molecular Cloning: a Laboratory Manual. Cold Spring Harbor, NY: Cold Spring Harbor Laboratory.

Mclafferty, M. A., Harcus, D. R., Weiss, A. A., Sapiain, L. A. \& HewletT, E. L. (1986). Development of a DNA probe for identification of Bordetella pertussis. Abstracts of the Annual Meeting of the American Society for Microbiology C168, 356.

MCPhEAT, W. L. \& MCNALLY, T. (1987). Isolation of a repeated DNA sequence from Bordetella pertussis. Journal of General Microbiology 133, 323-330.

Musser, J. M., Hewlett E. L., Peppler, M. S. \& SELANDER, R. K. (1986). Genetic diversity and relationships in populations of Bordetella spp. Journal of Bacteriology 166, 230-237. 
Nyman, K., Nakamura, K., Ohtsubo, H. \& Ohtsubo, E. (1981). Distribution of the insertion sequence IS1 in Gram-negative bacteria. Nature, London 289, 609-612.

RapPuoli, R., Perugini, M. \& Ratti, G. (1987). DNA element of Corynebacterium diptheriae with properties of an insertion sequence and usefulness for epidemiological studies. Journal of Bacteriology 169 , 308-312.

Rasmussen, J. L., Odelson, D. A. \& Macrina, F. L. (1987). Complete nucleotide sequence of insertion element IS4351 from Bacteroides fragilis. Journal of Bacteriology 169, 3573-3580.

Saesler, H., Reif, H. J., Hu, S. \& Davidson, N. (1974). IS2, a genetic element for turn-off and turnon of gene activity in E. coli. Molecular and General Genetics 132, 265-289.
Sanger, F., Nicklen, S. \& Coulson, A. R. (1977) DNA sequencing with chain-terminating inhibitors. Proceedings of the National Academy of Sciences of the United States of America 74, 5463-5467.

Scordilis, G. E., ReE, H. \& Lessie, T. G. (1987). Identification of transposable elements which activate gene expression in Pseudomonas cepacia. Journal of Bacteriology 169, 8-13.

SYVANEN, M. (1984). The evolutionary implications of mobile genetic elements. Annual Review of Genetics 18, 271-293.

Timmerman, K. P. \& Chen-Pei, D. T. (1985). Complete sequence of IS3. Nucleic Acids Research 13, 2127-2139.

YeE, T. \& INOUYe, M. (1981). Re-examination of the genome size of myxobacteria, including the use of a new method for genome size analysis. Journal of Bacteriology 145, 1257-1265. 